\title{
Localized Erythematous Pappular Rash: A Rare Presentation Of Essential Thrombocythemia
}

Mohd Shaiful Ehsa Shalihin ${ }^{1}$, Zulkifli Harun ${ }^{2}$, Iskandar Firzada Osman ${ }^{2}$

${ }^{1}$ Department of Family Medicine Specialist, Kulliyyah of Medicine, International Islamic University Malaysia

${ }^{2}$ Klinik Kesihatan Jaya Gading, Ministry of Health

Presenter: Mohd Shaiful Ehsan Shalihin

Essential thrombocythemia (ET) is one of the myeloproliferative neoplasms which typically presents with thrombotic vasomotor symptoms or bleeding tendencies. Papular rash is a rare manifestation of ET and may cause delay in making the diagnosis and hence institution of the treatment. We report a case of ET in a 50 year-old gentleman, who presented with nonspecific localized erythematous papular rash over his right thigh associated with recurrent mild pain in the affected thigh for one year. He was not any drugs prior to the onset of the rash. $\mathrm{He}$ had no history suggestive of haematological disorder such as bleeding tendencies, recurrent fever or anaemic symptoms. He also had no constitutional symptoms or any palpable masses. He was managed conservatively initially at primary care clinic but the problem persisted. A full blood count was subsequently performed which revealed an isolated thrombocytosis of $880 \times 10^{9} / \mathrm{L}$. He was referred to the haematology team for the further management and a diagnosis of ET was then made. Both the platelet counts and the lesion improved with hydroxyurea treatment. This case report illustrates a rare chronic manifestation of myeloproliferative neoplasm detected at a primary care clinic. 